\title{
Population Pharmacokinetic-Pharmacodynamic Relationships of Sarilumab Using Disease Activity Score 28-Joint C-Reactive Protein and Absolute Neutrophil Counts in Patients with Rheumatoid Arthritis
}

\author{
Lei Ma ${ }^{1} \cdot$ Christine $\mathrm{Xu}^{1} \cdot$ Anne Paccaly ${ }^{2} \cdot$ Vanaja Kanamaluru ${ }^{1}$
}

Published online: 26 May 2020

(c) The Author(s) 2020

\begin{abstract}
Background Sarilumab is a human monoclonal antibody blocking the interleukin-6 receptor alpha (IL-6Ra) approved for the treatment of moderately to severely active rheumatoid arthritis in adults with inadequate response or intolerance to other disease-modifying antirheumatic drugs.

Objective The aim of the current analysis was to describe sarilumab exposure-response relationships.

Methods Population pharmacokinetic/pharmacodynamic (PopPK/PD) models were developed describing the time course of the 28-joint disease activity score by C-reactive protein (DAS28-CRP) and absolute neutrophil count (ANC) using data from phase I-III studies (NCT01011959, NCT01061736, NCT01709578, NCT01768572) after subcutaneous sarilumab 50-150 mg every week or 100-200 mg every 2 weeks.

Results The time course of DAS28-CRP and ANC after sarilumab administration was described by semi-mechanistic, indirect-response models. Drug effect was predicted to be numerically greater at median exposure for the $200 \mathrm{mg}$ every 2 weeks regimen versus the $150 \mathrm{mg}$ every 2 weeks regimen, for both DAS28-CRP (50\% vs. 47\%) and ANC reduction from baseline (39\% vs. $31 \%$ ), with the latter showing less fluctuations within a dosing interval. Four covariates were retained in the final models: body weight, baseline rheumatoid factor status, anti-cyclic citrullinated peptide status, and concomitant methotrexate. There was no clinically meaningful influence of investigated covariates for either model.

Conclusion The PopPK/PD models showed numerically greater reductions in DAS28-CRP and ANC with sarilumab $200 \mathrm{mg}$ every 2 weeks than with $150 \mathrm{mg}$ every 2 weeks. There was no clinically meaningful influence of investigated covariates. These data contribute to the totality of evidence that supports a sarilumab subcutaneous starting dose of $200 \mathrm{mg}$ every 2 weeks, with a subsequent reduction to $150 \mathrm{mg}$ every 2 weeks in the event of laboratory abnormalities such as neutropenia.
\end{abstract}

\section{Introduction}

Rheumatoid arthritis (RA) is a chronic systemic autoimmune disease that can lead to irreversible joint damage, and is estimated to affect approximately 20 million people worldwide [1]. Interleukin (IL)-6 is a key multifunctional cytokine involved in the pathogenesis of inflammation and joint damage in RA [2]. The actions of IL-6 are mediated through classical cell signaling initiated through the membrane-bound IL-6 receptor alpha (IL-6Ra, the expression of which is limited to only a few cell types) and through

Christine Xu

christine.xu@sanofi.com

Sanofi Genzyme, Bridgewater, NJ, USA

Regeneron Pharmaceuticals, Inc, Tarrytown, NY, USA trans-signaling, whereby IL-6 binds to the soluble IL-6Ra, which then forms a signaling complex with glycoprotein 130 (gp130; which is expressed on most cell types). Inhibition of IL-6 signaling ameliorates the pathologic processes driven by persistently elevated IL-6 levels [2].

Sarilumab is a human immunoglobulin G1 (IgG1) monoclonal antibody that binds selectively to both membranebound and soluble IL-6Ra forms, blocking binding to the IL-6Ra and IL-6-mediated signaling [3-7]. Sarilumab is approved for the treatment of moderately to severely active $\mathrm{RA}$ in adult patients with an inadequate response (IR) to other disease-modifying antirheumatic drugs (DMARDs), as monotherapy or in combination with a conventional synthetic DMARD $[8,9]$. The approval of sarilumab was based on phase III studies that showed sarilumab rapidly improves signs and symptoms of RA (measured using a variety of clinical endpoints, including 28 -joint disease activity score 


\section{Key Points}

Using data from phase I-III studies among patients with rheumatoid arthritis treated with sarilumab, population pharmacokinetic/pharmacodynamic (PopPK/PD) models were developed to describe the time course of the 28 -joint disease activity score by $\mathrm{C}$-reactive protein (DAS28-CRP) and absolute neutrophil count (ANC).

The time course of both DAS28-CRP and ANC after sarilumab administration was described by semi-mechanistic, indirect-response models.

The PopPK/PD models showed numerically greater reductions in DAS28-CRP and ANC with sarilumab $200 \mathrm{mg}$ every 2 weeks than with $150 \mathrm{mg}$ every 2 weeks. These data contribute to the totality of evidence that support a sarilumab subcutaneous starting dose of $200 \mathrm{mg}$ every 2 weeks, with a subsequent reduction to $150 \mathrm{mg}$ every 2 weeks in the event of laboratory abnormalities (such as neutropenia).

Four covariates were retained in the final models: body weight, baseline rheumatoid factor status, anti-cyclic citrullinated peptide status, and concomitant methotrexate. None of these covariates had a clinically meaningful influence on the time course of DAS28-CRP or ANC during sarilumab treatment, indicating dose adjustments are not required for these patient demographics or disease characteristics.

by C-reactive protein [DAS28-CRP]), improves physical functioning, inhibits irreversible joint destruction, and is well tolerated [3-5]. Empirical exposure-response modeling showed a consistent trend toward improvement in clinical outcomes for subcutaneous sarilumab $200 \mathrm{mg}$ every 2 weeks compared with subcutaneous sarilumab $150 \mathrm{mg}$ every 2 weeks, including greater American College of Rheumatology (ACR) responses, and improvements in DAS28-CRP, Clinical Disease Activity Index (CDAI), Health Assessment Questionnaire Disability Index (HAQ-DI), and modified Total Sharp Score (mTSS) [10, 11].

In animals, IL-6 administration causes rapid mobilization of neutrophils from the marginated pool and release of neutrophils from the bone marrow into the blood circulation [12]. Consistent with this, IL-6Ra blockade has been associated with a reversible decrease in absolute neutrophil count (ANC). Reduced ANC is a potential safety concern. Although phase III studies were not powered to compare safety endpoints, the frequency and magnitude of ANC reductions were numerically greater with $200 \mathrm{mg}$ every 2 weeks compared with the lower $150 \mathrm{mg}$ every 2 weeks dose. However, clinical studies have shown that there is no relationship between the decrease in ANC observed during treatment with subcutaneous sarilumab 150 or $200 \mathrm{mg}$ every 2 weeks and the incidence of serious and non-serious infections [3-5, 13].

This paper reports the development of semi-mechanistic population pharmacokinetic/pharmacodynamic (PopPK/ PD) models that describe the relationship between drug concentration and change in DAS28-CRP (efficacy parameter) and ANC (safety parameter) over time following sarilumab administration in patients with RA, and identifies covariate factors influencing PK/PD relationships. This work was undertaken to corroborate the lack of need for dose adjustment based on patient demographics or baseline disease characteristics for adult patients with RA, and to add to the overall evidence to support sarilumab dose recommendations.

\section{Methods}

A sequential approach was used by first developing a PopPK model [14], followed by the development of a PopPK/PD model using individual sarilumab PK parameters derived from the PopPK model as the exposure input and the observed DAS28-CRP scores and ANC as clinical PD endpoints for efficacy and safety, respectively. PopPK parameters and individual estimates were computed assuming no effect of PD parameters on PK.

\subsection{Patients}

The previous PopPK model was developed based on PK data up to a predefined cut-off date from 1770 patients with RA who participated in phase I, II, and III studies [14]. For the current PopPK/PD analysis for DAS28-CRP, data up to the time of primary efficacy (disease activity) endpoint assessment (24 weeks) were pooled from 2082 patients (17,229 individual DAS28-CRP values) with RA enrolled in one phase II study (NCT01061736 Part A [MOBILITY]) and two phase III studies (NCT01061736 Part B [MOBILITY] and NCT01709578 [TARGET]) [Tables 1 and 2]. DAS28-CRP data were not available for the phase I, single-dose studies. Data for the PopPK/PD analysis for ANC were pooled from 1672 patients with RA enrolled in one phase I study (NCT01011959), one phase II study (NCT01061736 Part A [MOBILITY]), and three phase III studies (NCT01061736 Part B [MOBILITY], NCT01709578 [TARGET], and NCT01768572 [ASCERTAIN]) [Tables 1 and 2]. All clinical studies were performed in accordance with the principles of the Declaration of Helsinki and all applicable amendments by the World Medical Assemblies 
Table 1 Clinical studies included in the PopPK/PD analyses

\begin{tabular}{|c|c|c|c|}
\hline Clinical study identifier & Treatment doses and regimens ${ }^{\mathrm{a}}$ & Population & $\begin{array}{l}\text { Number of patients included in the PK/ } \\
\text { PD analyses }\end{array}$ \\
\hline \multicolumn{4}{|l|}{ PopPK/PD analysis for DAS28-CRP } \\
\hline NCT01061736 Part A (phase II) & $\begin{array}{l}\text { Placebo } \\
\text { Sarilumab } 100 \text { or } 150 \mathrm{mg} \mathrm{SC} \mathrm{qw} \\
\text { Sarilumab } 100,150 \text {, or } 200 \mathrm{mg}, \mathrm{SC} \\
\text { q2w } \\
\text { 12-week treatment duration }\end{array}$ & RA patients with MTX-IR & 254 (approximately 50 patients per arm) \\
\hline NCT01061736 Part B (phase III) & $\begin{array}{l}\text { Placebo } \\
\text { Sarilumab } 150 \text { or } 200 \mathrm{mg}, \mathrm{SC} \mathrm{q} 2 \mathrm{w} \\
\text { 52-week treatment duration }\end{array}$ & RA patients with MTX-IR & 1284 (approximately 350 patients/arm) \\
\hline NCT01709578 (phase III) & $\begin{array}{l}\text { Placebo } \\
\text { Sarilumab } 150 \text { or } 200 \mathrm{mg}, \mathrm{SC} \text { q2w } \\
\text { 24-week treatment duration }\end{array}$ & RA patients with TNF-IR & $\begin{array}{l}544 \text { (approximately } 174 \text { patients per } \\
\text { arm) }\end{array}$ \\
\hline \multicolumn{4}{|l|}{ PopPK/PD analysis for $A N C$} \\
\hline NCT01011959 (phase I) & $\begin{array}{l}\text { Placebo } \\
\text { Sarilumab 50, 100, or } 150 \mathrm{mg} \mathrm{SC} \mathrm{qw} \\
\text { Sarilumab 100, 150, or } 200 \mathrm{mg} \mathrm{SC} \\
\text { q2w } \\
\text { 5-week treatment duration }\end{array}$ & RA patients & 42 (7-8 patients/cohort) \\
\hline NCT01061736 Part A (phase II) & $\begin{array}{l}\text { Sarilumab } 100 \text { or } 150 \mathrm{mg} \mathrm{SC} \mathrm{qw} \\
\text { Sarilumab } 100,150 \text {, or } 200 \mathrm{mg}, \mathrm{SC} \\
\text { q2w } \\
\text { 52-week treatment duration }\end{array}$ & RA patients with MTX-IR & 203 (approximately 50 patients/arm) \\
\hline NCT01061736 Part B (phase III) & $\begin{array}{l}\text { Placebo } \\
\text { Sarilumab } 150 \text { or } 200 \mathrm{mg}, \mathrm{SC} \text { q2w } \\
\text { 24-week treatment duration }\end{array}$ & RA patients with MTX-IR & 968 (approximately 350 patients/arm) \\
\hline NCT01709578 (phase III) & $\begin{array}{l}\text { Placebo } \\
\text { Sarilumab 100, 150, or } 200 \mathrm{mg}, \mathrm{SC} \\
\text { q2w } \\
\text { 24-week treatment duration }\end{array}$ & RA patients with TNF-IR & 364 (approximately 174 patients/arm) \\
\hline NCT01768572 (phase III) ${ }^{\mathrm{c}}$ & $\begin{array}{l}\text { Sarilumab } 150 \text { or } 200 \mathrm{mg}, \mathrm{SC} q 2 \mathrm{w} \\
\text { 24-week treatment duration }\end{array}$ & RA patients with TNF-IR & $\begin{array}{l}95 \text { (sarilumab, approximately } 50 \\
\text { patients/arm; tocilizumab, approxi- } \\
\text { mately } 100 \text { patients) }\end{array}$ \\
\hline
\end{tabular}

$A N C$ absolute neutrophil count, DAS28-CRP 28-joint disease activity score by C-reactive protein, IR inadequate response, $M T X$ methotrexate, PopPK/PD population pharmacokinetic/pharmacodynamic, $q w$ weekly, $q 2 w$ every 2 weeks, $R A$ rheumatoid arthritis, $S C$ subcutaneous, $T N F$ tumor necrosis factor

${ }^{a}$ For placebo treatment, background RA therapy was administered; for active treatments, sarilumab was administered on top of background RA therapy

${ }^{b}$ Number of patients in the final data set

${ }^{\mathrm{c}}$ Study included a tocilizumab treatment arm; these data were not included in the current analysis

and the International Conference of Harmonisation Guidelines for Good Clinical Practice.

Patients treated with sarilumab for whom PK data were not available were excluded from the PopPK/PD analysis. Patients from NCT01709578 were not included in the PopPK model building as data from this study were not available by the predefined cut-off date for PopPK model building [14]. Therefore, the individual PK parameters for these patients were computed using an empirical Bayesian estimation approach (or maximum a posteriori).

\subsection{Clinical Endpoints}

The DAS28-CRP is a compositive score based on tender and swollen joints, serum levels of CRP, and the patient global health assessment, and was calculated at baseline and weeks 2 and 4, and then every 4 weeks across the three studies. ANC was derived as the sum of absolute counts of bands and absolute segmented neutrophils by multiplying the percentage of bands and segmented neutrophils by the total leukocyte count, and recorded at baseline, weeks 2, 4, 6, 8, 10 , and 12 , and then every 4 weeks across the five studies. 
Table 2 Demographic characteristics of the patients included in the final dataset for the development of the DAS28-CRP and ANC PopPK/ PD models

\begin{tabular}{lll}
\hline Covariate or characteristic & $\begin{array}{l}\text { PopPK/PD analysis for } \\
\text { DAS28-CRP } \\
\text { Final data set }(n=2082)\end{array}$ & $\begin{array}{l}\text { PopPK/PD analysis for ANC } \\
\text { Final data set }(n=1672)\end{array}$ \\
\hline Patient population MTX-IR/TNF-IR $[n(\%)]$ & $544(26.1) / 1538(73.9)$ & $459(27.5) / 1213(72.6)$ \\
Mean (SD) age, years & $51.6(12.0)$ & $51.7(12.1)$ \\
Mean (SD) body weight, kg & $74.6(18.8)$ & $74.1(18.7)$ \\
Mean baseline DAS28-CRP & - & $6.03(0.903)$ \\
Mean (SD) baseline IL-6, pg/mL & $41.1(64.5)$ \\
Mean (SD) baseline CRP, mg/L & $41.8(67.2)$ & $23.8(25.6)$ \\
Mean (SD) baseline PHYVAS & $24.1(25.1)$ & - \\
Mean (SD) baseline HAQ score & $64.6(16.8)$ & - \\
Female $[n(\%)]$ & $1.68(0.640)$ & $1374(82.2)$ \\
Caucasian $[n(\%)]$ & $1709(82.1)$ & $1418(84.8)$ \\
Smoker $[n(\%)]^{\mathrm{b}}$ & $1741(83.6)$ & $238(14.2)$ \\
MTX $[n(\%)]$ & $278(13.4)$ & $1634(97.7)$ \\
Baseline ACCP positive $[n(\%)]^{\mathrm{c}}$ & $2058(98.8)$ & $263(16.4)$ \\
Prior biologic treatment $[n(\%)]^{\mathrm{d}}$ & $337(16.5)$ & $591(38.5 \mathrm{l})$ \\
Prior corticosteroid treatment $[n(\%)]$ & $824(39.6)$ & $1067(63.8)$ \\
\hline
\end{tabular}

$A C C P$ anti-cyclic citrullinated peptide, ANC absolute neutrophil count, CRP C-reactive protein, DAS28CRP 28-joint disease activity score by C-reactive protein, $H A Q$ Health Assessment Questionnaire, IL-6 interleukin-6, IR inadequate responders, MTX methotrexate, PHYVAS Physician's Global Assessment of Disease Activity, PopPK/PD population pharmacokinetic/pharmacodynamic, $S D$, standard deviation, TNF, tumor necrosis factor

${ }^{a}$ DAS28-CRP model $n=1537$, ANC model $n=1213$; baseline IL-6 data were not available in study NCT01709578

${ }^{\mathrm{b}}$ DAS28-CRP model $n=2081$

${ }^{c}$ DAS28-CRP model $n=2047$, ANC model $n=1603$

${ }^{\mathrm{d}}$ ANC model $n=1535$

\subsection{Bioanalysis}

Concentrations of functional sarilumab in serum (i.e. sarilumab with one or two sites available for target binding, representing the pharmacologically active form), were measured using a validated immunoassay with a lower limit of quantification of $0.294 \mathrm{mg} / \mathrm{L}$ [14]. Immunogenicity was assessed using a validated, titer-based, bridging immunoassay. Samples that were positive in the antidrug antibody (ADA) assay (sensitivity based on the mouse monoclonal positive control was approximately $63.5-116.3 \mathrm{ng} / \mathrm{mL}$ ) were assessed for neutralizing antibodies (NAbs) using a validated competitive ligand binding assay (sensitivity based on the monoclonal positive control antibody, and the rabbit polyclonal antibody was approximately 150 and $257 \mathrm{ng} / \mathrm{mL}$, respectively).

\subsection{Model Building}

Indirect-response models were used to describe the time course of DAS28-CRP and ANC during treatment with sarilumab administered as subcutaneous doses of 50, 100, or $150 \mathrm{mg}$ every week, or 100,150 , or $200 \mathrm{mg}$ every 2 weeks using data pooled across studies, as shown in Table 1.
For the PopPK/PD analysis for both DAS28-CRP and ANC, the structural model was chosen on the basis of the examination of the objective function value (OFV), the inspection of conditional weighted residuals (CWRES) and individual weighted residuals (IWRES), the inspection of prediction and individual prediction versus observed plots, and visual inspection of the fits. Based on graphical evaluation (histograms and/or quantile-quantile plots of parameters), interindividual variability was modeled using a constant coefficient of variation or proportional error model (i.e. log-normal distribution). In modeling residual variability, errors between the observed and predicted PD (DAS28-CRP or ANC) values, $\varepsilon$, obtained using the individual's model predicted PD parameters, were assumed to arise from a symmetric distribution with a mean of zero and a variance of $\sigma^{2}$. Visual inspection of the distribution of CWRES versus population-predicted PD values and program output were used to assist in determining the structure of the residual variability model. The interindividual variability was evaluated for each model parameter.

The time course of DAS28-CRP following administration of subcutaneous sarilumab was best described by an indirect-response model linking sarilumab concentrations with 
DAS28-CRP via an inhibitory effect on the DAS28-CRP production rate $\left(k_{\text {in }}\right)$. The DAS28-CRP PopPK/PD model (Fig. 1) was parameterized with a first-order rate constant for the loss of response $\left(K_{\text {out }}\right)$, a maximum drug-induced effect $\left(E_{\max }\right)$, and a sarilumab concentration at $50 \%$ of maximum drug inhibitory effect $\left(\mathrm{IC}_{50}\right)$. The background treatment effect of concomitant DMARD therapies was included as a parameter in the model, termed PLB and expressed in sarilumab concentration units of $\mathrm{mg} / \mathrm{L}$.

The time course for ANC following subcutaneous administration of sarilumab was best described by an indirect-response model linking sarilumab concentrations with the ANC via stimulation of the elimination rate of neutrophils, i.e. disappearance from the bloodstream. The structure of the model was informed by the observation that IL-6R inhibition leads to neutrophil margination without loss of neutrophil function [19]. The ANC PopPK/PD model was parameterized with $K_{\text {out }}, E_{\text {max }}$, a sarilumab concentration for $50 \%$ of the maximum drug-induced effect $\left(\mathrm{EC}_{50}\right)$, and a Hill coefficient $(\gamma)$ (Fig. 1).

After the structural model selection, covariates were evaluated using a stepwise forward-addition and backward-deletion approach. Covariates providing a significant change $(p<0.001)$ in the OFV when introduced into the model were retained. A backward deletion was performed to assess the covariate-parameter relationship. Only covariates associated with a significant change in the OFV $(p<0.001)$ were retained in the final PopPK/PD models. The following covariates of interest were evaluated: demographics (sex, age, race, body weight, smoking status); biomarker (baseline CRP, baseline IL-6); disease status (baseline rheumatoid factor [RF], baseline anti-cyclic citrullinated peptide [ACCP] status, baseline DAS28-CRP, baseline HAQ-DI); medication (prior corticosteroid treatment, prior biologic treatment, concomitant methotrexate); patient population (methotrexateIR/DMARD-IR, tumor necrosis factor [TNF] therapy-IR); and ADA and NAb status (both positive or negative).

\subsection{Data Handling}

If any covariates were missing, the patient data were deleted from the analysis for categorical covariates and the missing value was computed from the population median

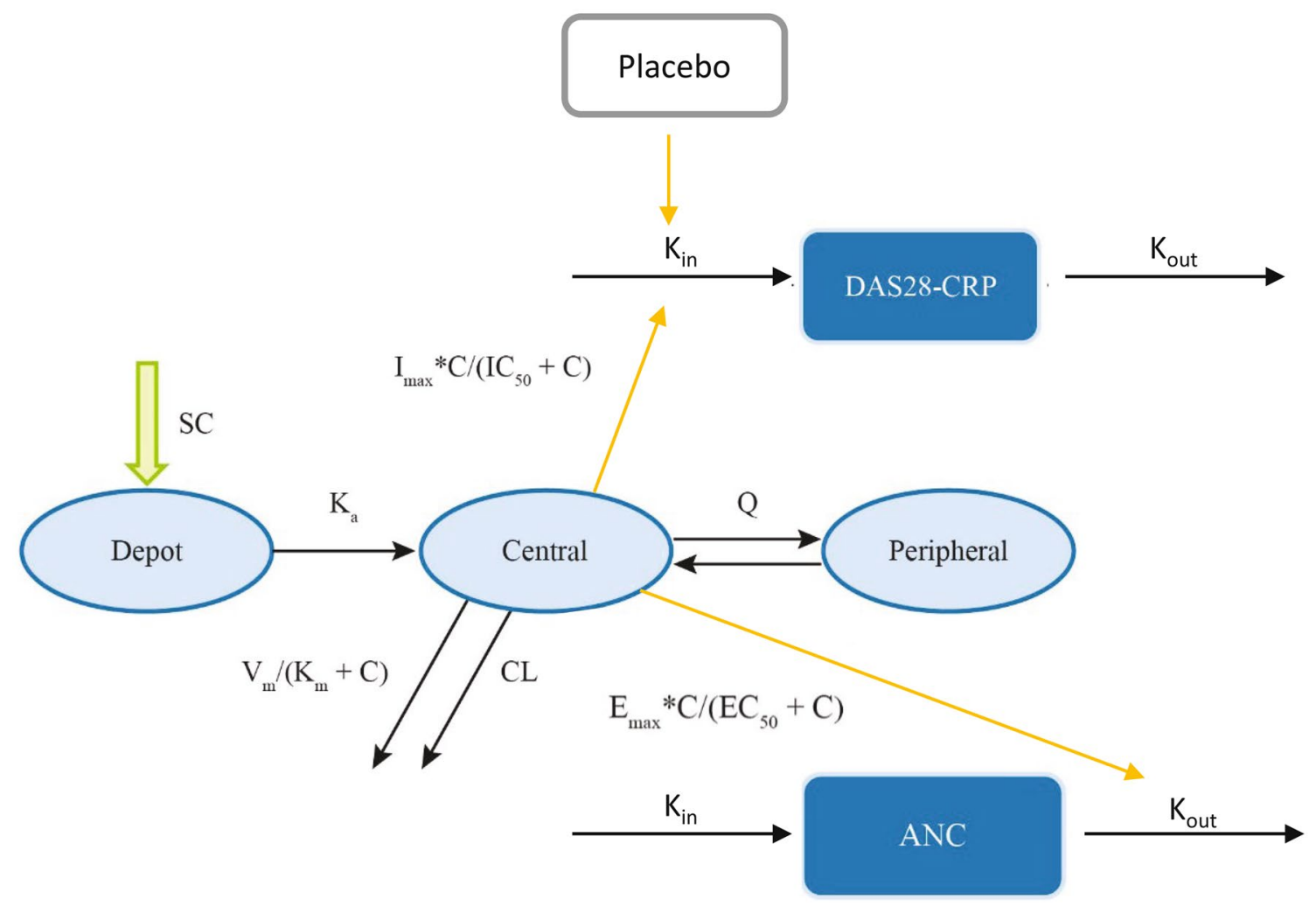

Fig. 1 DAS28-CRP and ANC PopPK/PD model structure. ANC absolute neutrophil count, $C$ concentration, $C L$ clearance, $D A S 28$ CRP 28-joint disease activity score by C-reactive protein, $\gamma$ Hill coefficient for sigmoidicity, $E C_{50}$ concentration at $50 \%$ of $E_{\max }, E_{\max }$ maximum drug effect, $I_{\max }$ maximum drug effect, $I C_{50}$ concentration at $50 \%$ of $I_{\max }, K_{a}$ first-order absorption rate constant, $K_{\text {in }}$ zero- order production rate, $K_{m}$ Michaelis-Menten constant, $K_{\text {out }}$ first-order degradation rate, $P o p P K / P D$ population pharmacokinetic/pharmacodynamic, $Q$ intercompartmental clearance, $S C$ subcutaneous, $V_{m}$ maximum rate. DAS28-CRP model: $\operatorname{Eff}(C)=E_{\max } \times(C+\text { placebo })^{\gamma /}$ $\left(\mathrm{IC}_{50}{ }^{\gamma}+(C+\text { placebo })^{\gamma}\right)$. ANC model: $\operatorname{Eff}(C)=E_{\max } \times C^{\gamma} /\left(\mathrm{EC}_{50}{ }^{\gamma}+C^{\gamma}\right)$ 
for continuous covariates. If a strong correlation existed between any other covariates, a linear regression could be used to calculate the covariate value. As all covariates were tested at baseline, there was no need to carry observations forward or back for missing values.

For any covariate data that were completely unavailable for any individual study, or any categorical covariate that was $<10 \%$ of the total included patients, a post hoc approach was employed that used descriptive statistics of the individual model parameters by covariate category and plot of individual values of model parameters versus covariates (box plots for categorical covariates and scatter plots for continuous covariates). Since baseline IL-6 data were not available in the NCT01709578 and NCT01768572 studies, and NAb data were not available in phase I and II studies, a post hoc approach was used for these covariates. It was assumed that the distributions of other covariates across the different levels within that covariate were approximately balanced.

\subsection{Model Evaluation}

The final PopPK/PD models were verified by evaluation of goodness-of-fit plots, visual predictive check (VPC), and bootstrap analyses. Bootstrap analyses were conducted for DAS28-CRP and ANC using 1000 bootstrap sets drawn with replacement (resampling) from the original datasets for the respective analysis. For each of the 1000 bootstrap sets, the population PD parameters were estimated and the corresponding mean, median, standard deviation (SD), and 2.5th/97.5th percentiles were calculated. To validate each PopPK/PD model, the mean parameter estimates obtained in the final model of the original dataset were compared with median parameters from bootstraps. Analyses were performed using NONMEM ${ }^{\circledR}$ version 7.2 (ICON plc, Dublin, Ireland) running on a Linux cluster of multiprocessor computers. All runs were performed using the first-order conditional estimate method with interaction option.

\section{Results}

\subsection{8-Joint Disease Activity Score by C-Reactive Protein (DAS28-CRP) Population Pharmacokinetic/Pharmacodynamic (PopPK/ PD) Model}

The final PopPK/PD model parameter estimates translated into a 55.9\% maximum decrease in DAS28-CRP score from baseline, with an $\mathrm{IC}_{50}$ of $2.32 \mathrm{mg} / \mathrm{L}$ (Table 3), and included four covariates on baseline DAS28-CRP (baseline
CRP level, baseline physician's global assessment of disease activity, baseline HAQ-DI, and body weight), one covariate on $E_{\max }$ (baseline CRP level), and one covariate on $K_{\text {out }}$ (corticosteroid treatment at baseline).

DAS28-CRP baseline values and $E_{\max }$ decreased with a decrease in baseline CRP. DAS28-CRP baseline values were $6.6 \%$ lower in patients with a baseline CRP of $8.17 \mathrm{mg} / \mathrm{L}$ (25th percentile) and $7.5 \%$ higher in patients with a baseline CRP of $31.2 \mathrm{mg} / \mathrm{L}$ (75th percentile), compared with patients with a baseline CRP of $15.7 \mathrm{mg} / \mathrm{L}$ (median); $E_{\max }$ was $0.7 \%$ lower and $0.8 \%$ higher, respectively. DAS28-CRP baseline values also decreased with a decrease in baseline physicians global assessment score $(1.1 \%$ lower with a score of 54 and $0.9 \%$ higher with a score of 77 , compared with a median score of 66), a decrease in baseline HAQ-DI score (3.9\% lower with a score of 1.25 and $2.9 \%$ higher with a score of 2.13 , compared with a median score of 1.75), and a decrease in body weight $(0.9 \%$ lower in those weighing $61 \mathrm{~kg}$ and $0.8 \%$ higher in those weighing $84.3 \mathrm{~kg}$, compared with a body weight of $72.8 \mathrm{~kg}$ ). The $K_{\text {out }}$ value was $26 \%$ higher in patients with prior corticosteroid treatment compared with those without $\left(0.0333\right.$ vs. 0.0264 day $\left.^{-1}\right)$, resulting in minimal DAS28-CRP changes $(<1 \%)$ at week 24 . Thus, the effect of these covariates on the time course of DAS28-CRP was small and not clinically meaningful.

Sex, age, race, baseline RF, baseline ACCP status, prior treatment with biologics, concomitant methotrexate, or patient population (methotrexate or TNF $\alpha$ antagonist IRs) were not identified as significant covariates in the final PopPK/PD model. Additionally, (post hoc) baseline IL-6 level, ADA status, and NAb status had no appreciable impact.

\subsubsection{DAS28-CRP PopPK/PD Model Predicted Versus Observed Evaluation}

The goodness-of-fit, CWRES, and normalized prediction distribution error (NPDE) evaluation results (Fig. 2a-c) indicate that the final DAS28-CRP PopPK/PD model was consistent with the observed data, except for the cohort of patients initially randomized to placebo who subsequently received sarilumab $200 \mathrm{mg}$ every 2 weeks. The VPC (Fig. 2d) and bootstrap analysis (Table 3) showed that simulated DAS28-CRP time profiles (2.5th, 50th, and 97.5th percentiles) fitted the observed data well.

The effect of sarilumab on DAS28-CRP was predicted to be $50.3 \%$ at the median trough concentration of the $200 \mathrm{mg}$ every 2 weeks regimen and $46.5 \%$ at the median trough concentration of the $150 \mathrm{mg}$ every 2 weeks regimen (Fig. 3). At week 24, the model-estimated mean (SD) DAS28-CRP was 3.19 (1.05), compared with an observed value of 3.24 
Table 3 Parameter estimates for the DAS28-CRP PopPK/PD model

\begin{tabular}{|c|c|c|c|c|}
\hline \multirow[t]{2}{*}{ Parameter } & \multicolumn{3}{|c|}{ Final model population mean } & \multirow{2}{*}{$\begin{array}{l}\text { Bootstrap estimate } \\
\text { Median }(95 \% \mathrm{CI})\end{array}$} \\
\hline & Estimate & $\%$ RSE & $95 \% \mathrm{CI}$ & \\
\hline BASE & 6.06 & 0.32 & $6.03-6.10$ & $6.07(6.03-6.10)$ \\
\hline $\log \left(E_{\max }\right)$ & 0.237 & 19.8 & $0.143-0.331$ & $0.246(0.157-0.384)$ \\
\hline $\mathrm{IC}_{50}, \mathrm{mg} / \mathrm{L}$ & 2.32 & 17.6 & $1.51-3.14$ & $2.41(1.74-3.29)$ \\
\hline$K_{\text {out }}$, day $^{-1}$ & 0.0264 & 4.61 & $0.0239-0.0289$ & $0.0264(0.0233-0.0301)$ \\
\hline$\gamma$ & 1 (fixed) & NA & NA & \\
\hline PLB, mg/L & 0.991 & 19.1 & $0.612-1.37$ & $1.03(0.737-1.40)$ \\
\hline BLCRP on BASE & 0.0564 & 4.49 & $0.0513-0.0614$ & $0.0563(0.0514-0.0614)$ \\
\hline BLPHYVAS on BASE & 0.105 & 6.41 & $0.0913-0.118$ & $0.105(0.0834-0.127)$ \\
\hline BLHAQ on BASE & 0.0779 & 5.15 & $0.0689-0.0859$ & $0.0778(0.0695-0.0858)$ \\
\hline Weight on BASE & 0.0522 & 20.2 & $0.0311-0.0733$ & $0.0512(0.0305-0.0738)$ \\
\hline BLCRP on $\log \left(E_{\max }\right)$ & 0.333 & 34.5 & $0.103-0.562$ & $0.321(0.154-0.535)$ \\
\hline \multirow[t]{3}{*}{ PRICORT on $K_{\text {out }}$} & 1.26 & 5.50 & $1.12-1.40$ & $1.27(1.10-1.48)$ \\
\hline & \multicolumn{3}{|c|}{ Final model interindividual variability (\%) } & $\begin{array}{l}\text { Bootstrap interindividual } \\
\text { variability }(\%)\end{array}$ \\
\hline & Estimate & $\% \mathrm{RSE}$ & Shrinkage $(\%)$ & $8.03(7.48-8.54)$ \\
\hline BASE & 8.05 & 6.86 & 31.3 & 70.8 (63.5-93.5) \\
\hline $\log \left(E_{\max }\right)$ & 71.2 & 8.26 & 40.5 & $157(44.2-183)$ \\
\hline $\mathrm{IC}_{50}, \mathrm{mg} / \mathrm{L}$ & 158 & 13.4 & 42.3 & $84.2(78.1-92.3)$ \\
\hline$K_{\text {out }}$, day $^{-1}$ & 84.2 & 6.11 & 31.9 & $107(49.4-161)$ \\
\hline $\mathrm{PLB}, \mathrm{mg} / \mathrm{L}$ & 105 & 38.1 & 75.8 & $107(49.4-161)$ \\
\hline \multicolumn{5}{|l|}{ Residual variability } \\
\hline Additive term, mg/L & 0.647 & 0.70 & 11.0 & $0.646(0.632-0.662)$ \\
\hline
\end{tabular}

$B A S E$ baseline, BLCRP baseline C-reactive protein, BLHAQ baseline Health Assessment Questionnaire, BLPHYVAS baseline Physician's Global Assessment of Disease Activity, CI confidence interval, DAS28-CRP 28-joint disease activity score by C-reactive protein, DMARD diseasemodifying antirheumatic drug, $E_{\max }$ maximum drug-induced effect, $\gamma$ hill coefficient for sigmoidicity, $I C_{50}$ concentration of $50 \%$ of $E_{\max }, K_{\text {out }}$ first-order rate constant, $\log \left(E_{\max }\right) \log$-transformed maximum drug effect, $N A$ not applicable, $P L B$ placebo/background treatment effect of concomitant DMARDs in sarilumab concentration units, PopPK/PD population pharmacokinetic/pharmacodynamic, $P R I C O R T$ prior corticosteroid treatment, \%RSE relative standard error (100\%*SE/estimate), $S E$ standard error

(1.30) for sarilumab $150 \mathrm{mg}$ every 2 weeks and 3.01 (1.02) versus 3.01 (1.25) for sarilumab $200 \mathrm{mg}$ every 2 weeks, respectively. Compared with a $150 \mathrm{mg}$ every 2 weeks dose, a greater proportion of patients at $200 \mathrm{mg}$ every 2 weeks maintained sarilumab trough concentrations above that needed for half maximal effect for DAS28-CRP (82\% vs. 54\%).

\subsection{Absolute Neutrophil Count (ANC) PopPK/PD Model}

The final PopPK/PD model parameter estimates translated into a maximal decrease in circulating ANC from baseline of $60 \%$ and the lowest possible ANC level of $2.15 \times 10^{9} / \mathrm{L}$, with an $\mathrm{IC}_{50}$ of $10.3 \mathrm{mg} / \mathrm{L}$ (Table 4). The model included one covariate on baseline ANC (smoking status), one covariate on $E_{\max }$ (baseline corticosteroid treatment), and one covariate on $K_{\text {out }}$ (body weight). The baseline ANC value was $15.1 \%$ higher in patients who were smokers than in patients who were non-smokers $\left(6.19\right.$ vs. $\left.5.38 \times 10^{9} / \mathrm{L}\right)$. The $E_{\max }$ value was $18.1 \%$ lower in patients with prior corticosteroid treatment than patients without prior corticosteroid treatment (1.23 vs. 1.50), translating into a $55 \%$ and $60 \%$ maximal decrease of ANC in patients with and without prior corticosteroid treatment, respectively. Additionally, compared with patients with a body weight of $71 \mathrm{~kg}$ (median), the $K_{\text {out }}$ value was $12.4 \%$ lower in patients with a body weight of $61.0 \mathrm{~kg}$ (25th percentile) and $15.7 \%$ higher in those with a body weight of $83.9 \mathrm{~kg}$ (75th percentile). However, this did not translate to a meaningful change in ANC $(<1 \%)$ at week 24 . Thus, the effect of these covariates on the PD parameters was small, with none having a clinically meaningful influence on ANC. 
Sex, age, race, baseline RF, baseline ACCP status, baseline DAS28-CRP, prior treatment with biologics, concomitant methotrexate treatment, or patient population (methotrexate or TNF $\alpha$ antagonist IRs) were not identified as significant covariates in the final PopPK/PD model. Additionally, (post hoc) baseline IL-6 level, ADA status, and NAb status had no appreciable impact.

\subsubsection{ANC PopPK/PD Model Predicted Versus Observed Evaluation}

The goodness-of-fit, CWRES, and NPDE evaluation results (Fig. 4a-c) indicate that the final ANC PopPK/PD model was consistent with the observed data, except for the cohort of patients initially randomized to placebo who subsequently received sarilumab $200 \mathrm{mg}$ every 2 weeks. The results of the VPC (Fig. 4d) and bootstrap analysis (Table 4) showed that simulated ANC time profiles(2.5th, 50th, and 97.5th percentiles) track observed trends very well, especially for the $150 \mathrm{mg}$ every 2 weeks and $200 \mathrm{mg}$ every 2 weeks regimens and represent a good qualification of the PopPK/PD model developed in the present analysis.

Within a dose interval, ANC decreased to the nadir and recovered toward baseline in a dose- and concentrationdependent manner, with higher fluctuations for the $150 \mathrm{mg}$ every 2 weeks dose regimen than for the $200 \mathrm{mg}$ every 2 weeks regimen (Fig. 5). Compared with a $150 \mathrm{mg}$ every 2 weeks dose, a greater proportion of patients at $200 \mathrm{mg}$ every 2 weeks maintained sarilumab trough concentrations above that needed for half maximal effect for $\mathrm{ANC}$ reduction (60\% vs. $28 \%$ ). The reduction in the ANC from baseline was predicted to be slightly higher at the median trough concentration of $200 \mathrm{mg}$ every 2 weeks (39\%) than the median trough concentration of $150 \mathrm{mg}$ every 2 weeks (31\%) at steady state. At week 24, the model-estimated mean (SD) ANC was 4.10 (1.94), compared with an observed value of 4.24 (2.31) for sarilumab $150 \mathrm{mg}$ every 2 weeks and 3.68 (1.83) versus 3.79 (2.19) for sarilumab $200 \mathrm{mg}$ every 2 weeks, respectively.

\section{Discussion}

The time course observed in patients for DAS28-CRP and ANC following sarilumab subcutaneous administration was well described, with indirect-response population PopPK/PD models linking concentrations of sarilumab with DAS28-CRP via an inhibitory effect on the 'production rate' of DAS28-CRP $\left(K_{\text {in }}\right)$, or with ANC via stimulation of the 'elimination rate' of neutrophils $\left(K_{\text {out }}\right)$. The DAS28-CRP model is consistent with the mode of action of sarilumab in
Fig. 2 DAS28-CRP PopPK/PD model. a Goodness-of-fit analysis; b normalized prediction distribution error; c conditional weighted residuals; d visual predictive check. CWRES conditional weighted residuals, $D A S 28$-CRP 28-joint disease activity score by C-reactive protein, $N P D E$ normalized prediction distribution error, PopPK/PD population pharmacokinetic/pharmacodynamic, $q w$ every week, $q 2 w$ every 2 weeks. In a, dashed blue line indicates $y=x$, solid blue line indicates $y=0$, solid red line indicates tendency. In $\mathbf{d}$, solid red line represents the median of observations; solid dashed lines represent the 2.5th and 97.5th percentiles of observations; and pink and gray areas represent the confidence intervals of the median and $5^{\text {th }}$ and 95th percentiles of predictions. Placebo $+200 \mathrm{mg} \mathrm{q} 2 \mathrm{w}$ defines the cohort of patients initially randomized to placebo who subsequently received sarilumab $200 \mathrm{mg} \mathrm{q} 2 \mathrm{w}$

terms of inhibition of disease progression $\left(K_{\text {in }}\right)$. Similarly, the ANC model is consistent with the postulated 'margination' of functioning neutrophils from the circulation, the central compartment $\left(K_{\text {out }}\right)[15,16]$. For DAS28-CRP, the final model included four covariates on baseline DAS28-CRP (baseline CRP level, baseline physician's global assessment of disease activity, baseline HAQ-DI, and body weight), one covariate on $E_{\max }$ (baseline CRP level), and one covariate on $K_{\text {out }}$ (corticosteroid treatment at baseline). The final ANC PopPK/PD model included one covariate of baseline ANC (smoking status), one covariate of $E_{\max }$ (baseline corticosteroid treatment), and one covariate of $K_{\text {out }}$ (body weight). None of the identified covariates had a clinically meaningful influence on the time course of DAS28-CRP or ANC during sarilumab treatment, indicating dose adjustments are not required for these patient characteristics. These observations are consistent with the PopPK analysis described previously [14], where none of the covariates evaluated, including body weight, had any clinically meaningful effect on the PK of sarilumab.

Simulated time profiles validated the predictive ability of the PopPK/PD models. For DAS28-CRP, there was a slight underprediction at the later time points ( $>24$ weeks) for the sarilumab treatment groups. For the placebo group, the simulated values generally underpredicted the observed values (except for values derived from patients in the placebo group who rescued with open-label sarilumab $200 \mathrm{mg}$ every 2 weeks from week 12 onwards in the case of lack of efficacy [per protocol], which were overpredicted).

Efficacy results from phase III clinical studies favored a $200 \mathrm{mg}$ every 2 weeks dosing regimen over the $150 \mathrm{mg}$ every 2 weeks regimen [3-5]. In TARGET (patients with moderately to severely active RA who had an IR or intolerance to anti-TNF therapy), the mean change from baseline in DAS28-CRP score at week 24 was - 2.4 (standard error 0.11) for patients treated with sarilumab $150 \mathrm{mg}$ and $-2.8(0.11)$ for those treated with sarilumab $200 \mathrm{mg}$ every 2 weeks. The proportion of patients achieving DAS28-CRP responses 
(a)
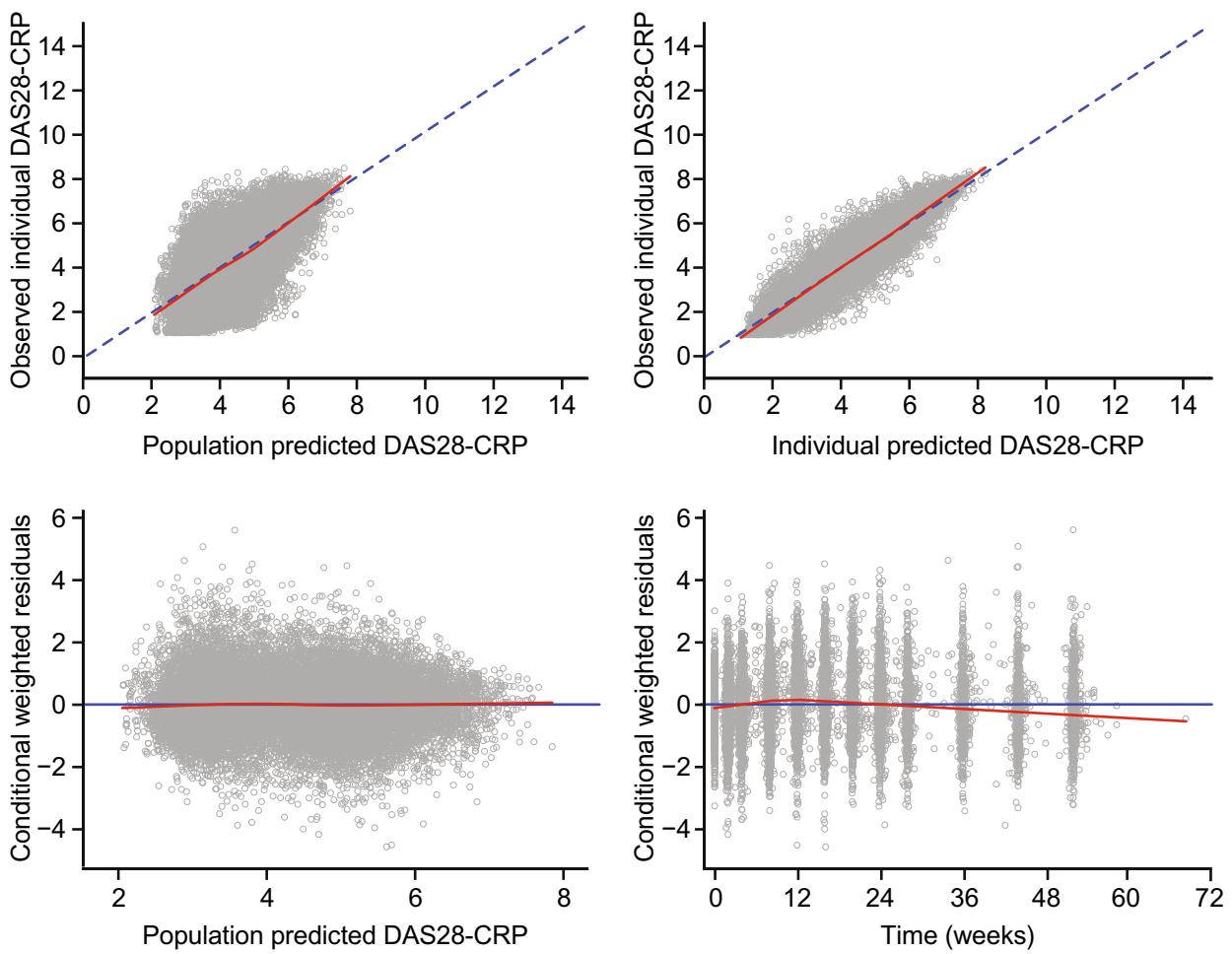

(b)
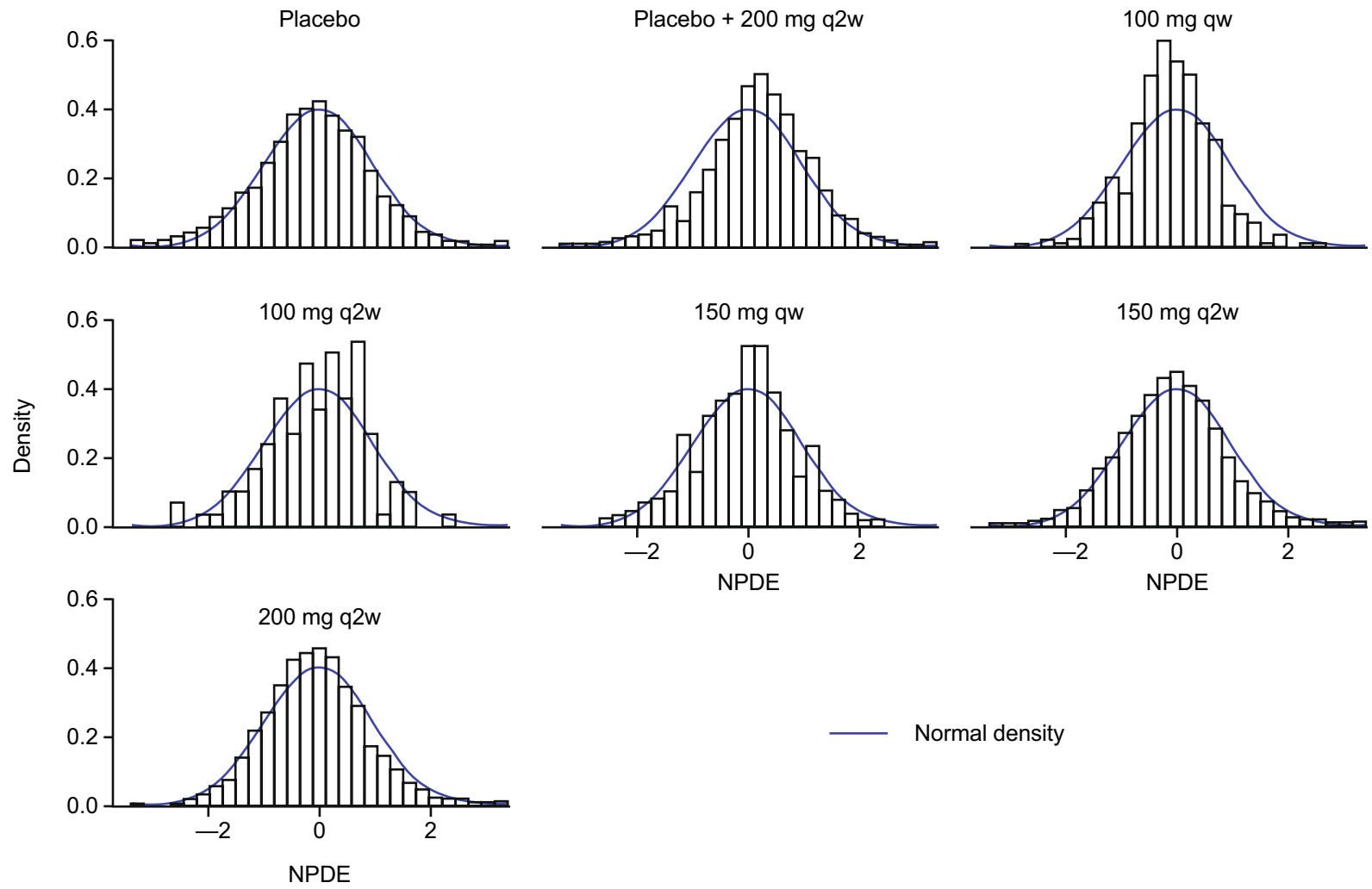

Normal density 
(c)
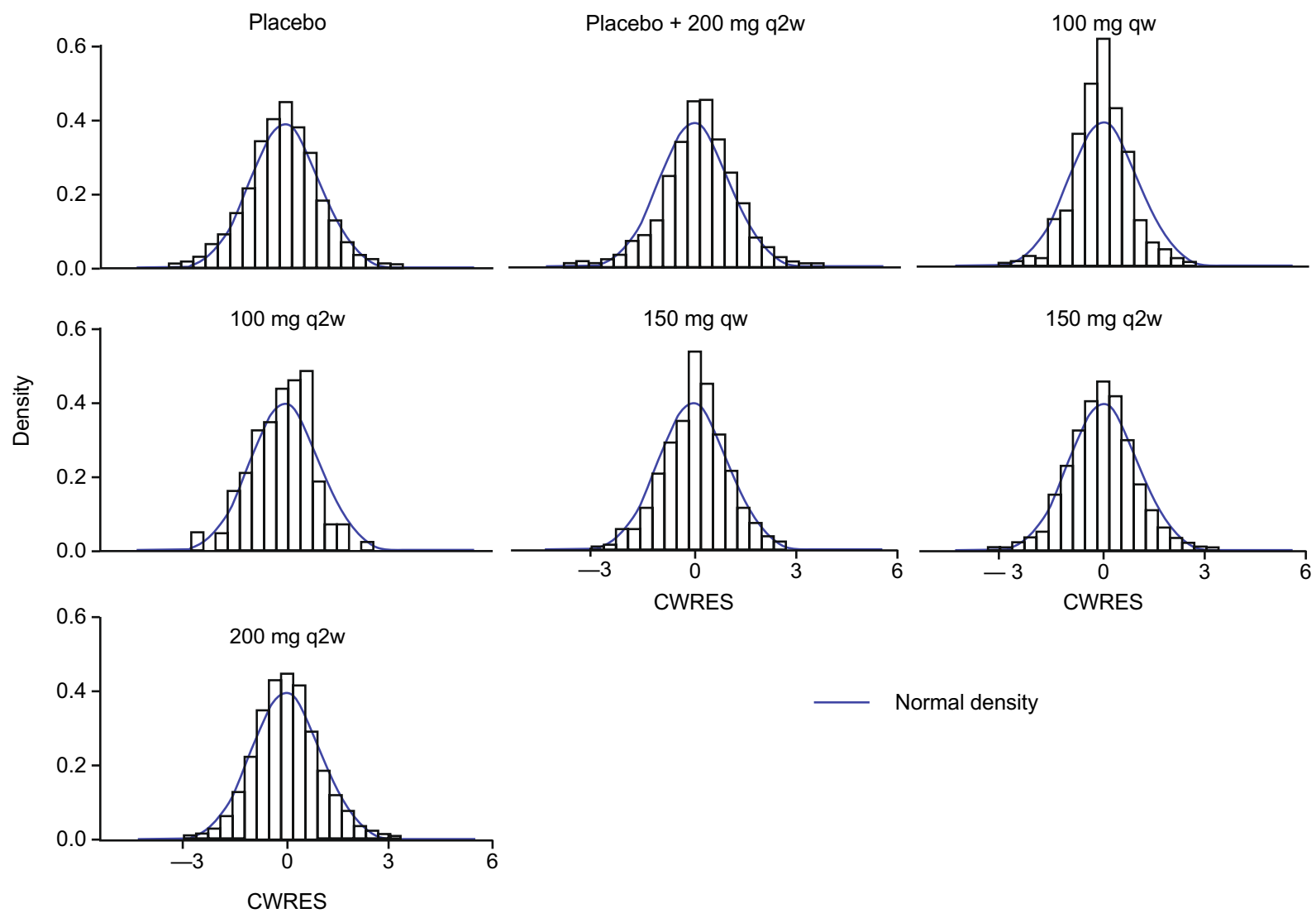

Normal density

(d)

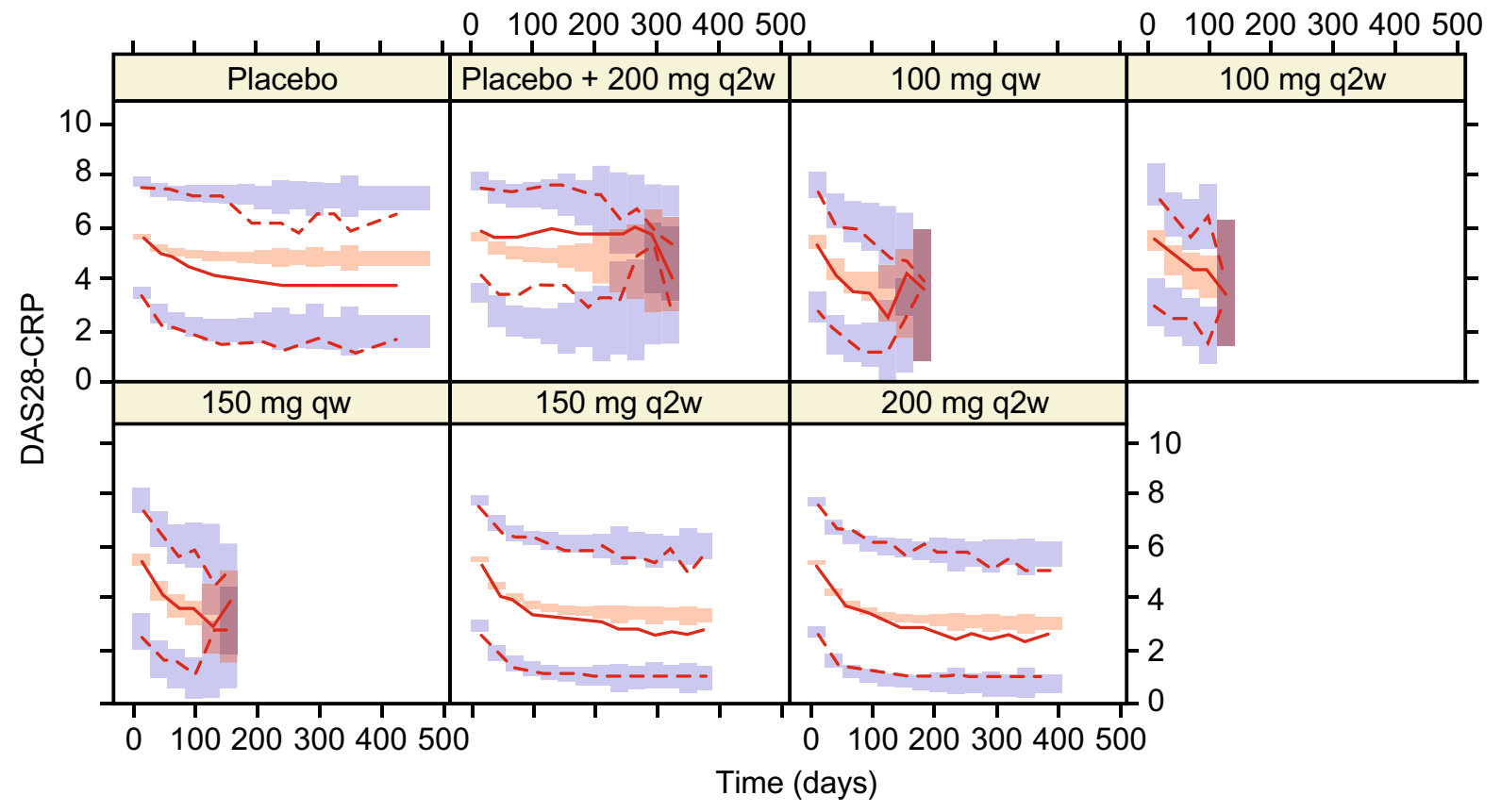

Fig. 2 (continued) 
Fig. 3 DAS28-CRP time profiles (observed vs. modelpredicted). DAS28-CRP 28-joint disease activity score by C-reactive protein, $P 55$ th percentile, $P 9595$ th percentile, $q 2 w$ every 2 weeks

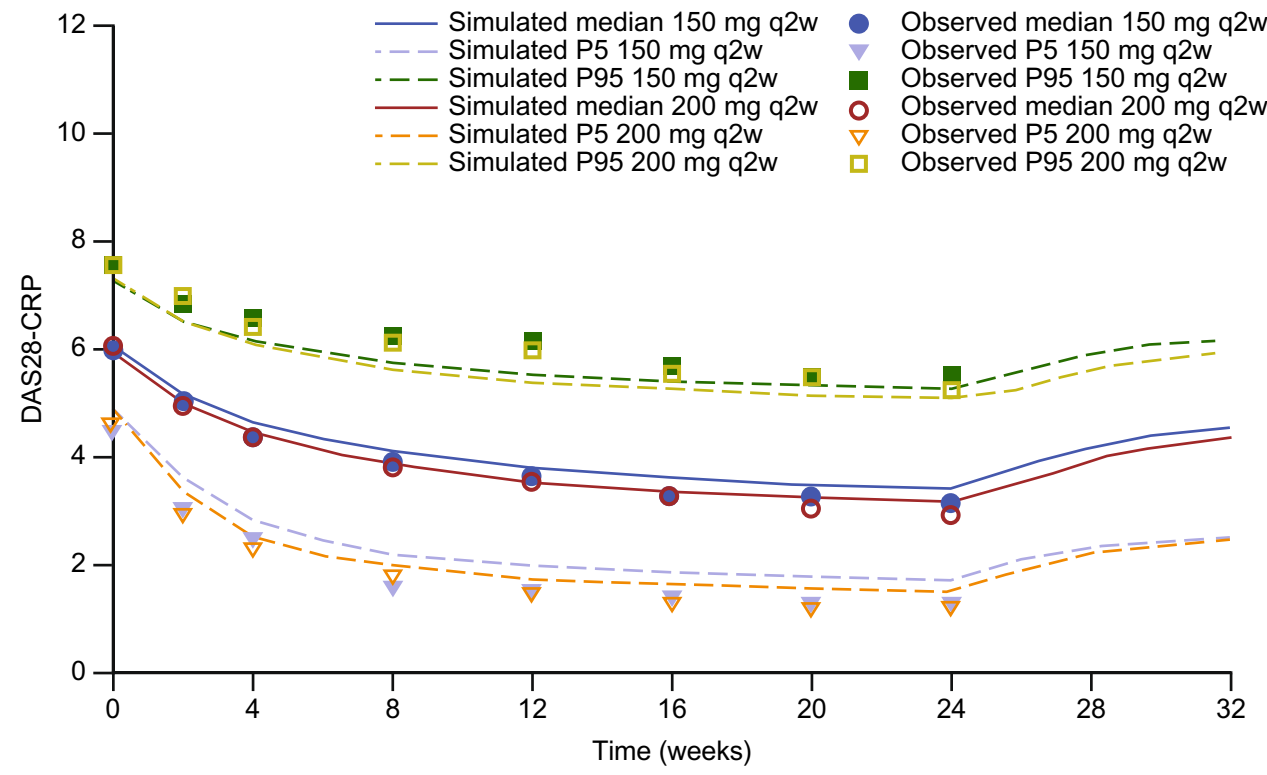

Table 4 Parameter estimates for the ANC PopPK/PD model

\begin{tabular}{|c|c|c|c|c|}
\hline \multirow[t]{2}{*}{ Parameter } & \multicolumn{3}{|c|}{ Population mean } & \multirow{2}{*}{$\begin{array}{l}\text { Bootstrap estimate } \\
\text { Median (95\% CI) }\end{array}$} \\
\hline & Estimate & $\%$ RSE & $95 \% \mathrm{CI}$ & \\
\hline BASE & 5.38 & 1.05 & $5.27-5.50$ & $5.37(5.26-5.51)$ \\
\hline$E_{\max }$ & 1.50 & 4.62 & $1.36-1.64$ & $1.44(1.26-1.72)$ \\
\hline$E C_{50}, \mathrm{mg} / \mathrm{L}$ & 10.3 & 6.16 & $9.00-11.5$ & $10.3(7.53-14.1)$ \\
\hline$K_{\text {out }}$, day $^{-1}$ & 2.17 & 35.3 & $0.638-3.71$ & $211(1.67-2.88)$ \\
\hline$\Gamma$ & 0.862 & 4.55 & $0.783-0.940$ & $0.840(0.747-0.961)$ \\
\hline Smoking on BASE ${ }^{\mathrm{a}}$ & 1.15 & 2.64 & $1.09-1.21$ & $1.16(1.10-1.21)$ \\
\hline Weight $^{\mathrm{b}}$ on $K_{\text {out }}^{\mathrm{a}}$ & 0.875 & 12.2 & $0.662-1.09$ & $0.866(0.219-0.964)$ \\
\hline \multirow[t]{3}{*}{ PRICORT on $E_{\max }{ }^{\text {a }}$} & 0.819 & 4.63 & $0.743-0.895$ & $0.850(0.755-0.948)$ \\
\hline & \multicolumn{3}{|c|}{ Interindividual variability (\%) } & $\begin{array}{l}\text { Bootstrap interin- } \\
\text { dividual variability } \\
(\%)\end{array}$ \\
\hline & Estimate & $\%$ RSE & Shrinkage (\%) & Median $(95 \%$ CI) \\
\hline BASE & 32.1 & 4.61 & 12.9 & $32.1(30.5-33.5)$ \\
\hline$E_{\max }$ & 61.9 & 6.83 & 30.6 & $61.2(54.9-67.9)$ \\
\hline $\mathrm{EC}_{50}, \mathrm{mg} / \mathrm{L}$ & 36.9 & 28.0 & 74.0 & $39.5(21.1-51.0)$ \\
\hline$K_{\text {out }}$, day $^{-1}$ & 227 & 43.7 & 79.3 & $232(198-279)$ \\
\hline$\Gamma$ & 80.4 & 8.89 & 42.3 & $83.2(63.8-101)$ \\
\hline \multicolumn{5}{|l|}{ Residual variability } \\
\hline Proportional term (\%) & 28.2 & 0.8 & 7.14 & $28.3(27.6-28.9)$ \\
\hline
\end{tabular}

$A N C$ absolute neutrophil count, $B A S E$ baseline ANC, $C I$ confidence interval, $E C_{50}$ concentration at $50 \%$ of $E_{\max }, E_{\max }$ maximum drug-induced effect, $\gamma$ Hill coefficient for sigmoidicity, $K_{\text {out }}$ first-order rate constant for loss of ANC rate, PopPK/PD population pharmacokinetic/pharmacodynamic, PRICORT prior corticosteroid treatment, $\% R S E$ relative standard error (100\%*SE/estimate), SE standard error

${ }^{\mathrm{a}}$ Covariates included in the final PopK/PD model as a power function

${ }^{\mathrm{b}}$ Expressed relative to a median weight of $71 \mathrm{~kg}$ 
of $<2.6$ and $\leq 3.2$ at week 24 was $24.9 \%$ and $32.6 \%$, respectively, in the sarilumab $150 \mathrm{mg}$ every 2 weeks group, and $28.8 \%$ and $40.2 \%$, respectively, in the $200 \mathrm{mg}$ every 2 weeks group [3]. Similarly, in MOBILITY, conducted in methotrexate-IR patients with moderate to severe RA, the proportion of patients achieving DAS28-CRP responses of $<2.6$ and $\leq 3.2$ at week 24 was $27.8 \%$ and $39.8 \%$, respectively, in the sarilumab $150 \mathrm{mg}$ every 2 weeks group, and $34.1 \%$ and $49.1 \%$ in the $200 \mathrm{mg}$ every 2 weeks group [4]. In the latter study, the proportion of patients without radiographic progression of structural joint damage, which is irreversible, at week 24 was higher among those patients treated with sarilumab $200 \mathrm{mg}$ every 2 weeks (56.6\%) than among those patients treated with $150 \mathrm{mg}$ every 2 weeks (46.3\%) [3-5]. Consistent with these clinical observations, the PopPK/PD model showed a numerically greater reduction in DAS28CRP at the median trough concentration for the 200 versus $150 \mathrm{mg}$ every 2 weeks regimens.

Clinical studies have also shown that sarilumab is associated with a decrease in ANC, which is reversible and manageable by dose adjustment [3-5]. Consistent with this, the ANC time course was described by an indirect-response model with a rapid decrease within 1-2 weeks of initiating subcutaneous sarilumab (200 and $150 \mathrm{mg}$ every 2 weeks), with ANC levels stabilizing after 4 weeks of treatment. This PopPK/PD analysis showed that the $150 \mathrm{mg}$ every 2 weeks regimen would result in a marginally smaller reduction in ANC and a more rapid return toward baseline compared with the $200 \mathrm{mg}$ every 2 weeks regimen. The observed effect on $K_{\text {out }}$ for sarilumab can be explained by margination of neutrophils from the vascular circulation to the vascular wall or other tissue [17]. A reversible decrease in ANC has also been reported for another IL-6 receptor blocker, tocilizumab, indicating that this phenomenon is a consequence of inhibition of IL-6 signaling $[15,16]$. A previous analysis has indicated that the pharmacological effect on ANC levels for both subcutaneous sarilumab and intravenous tocilizumab is immediate and relatively short-lasting after a single dose with an apparent saturation of effect and pharmacological tolerance [13]. The apparent saturation of effect is evidenced by the temporal PopPK/PD relationship for ANC versus sarilumab concentrations, with ANC values returning toward baseline even as sarilumab concentrations continued to increase. After a single $200 \mathrm{mg}$ dose of subcutaneous sarilumab, a return to baseline in ANC was achieved at around 2 weeks postdose. A return toward baseline ANC was also noted for intravenous tocilizumab, with baseline levels achieved at around 1 month following a single intravenous dose. However, regardless of the decrease in ANC, no relationship between decrease in ANC and infection, including serious infection, has been identified in clinical trials for sarilumab, indicating that neutrophils remain available and functioning in case of an infection and can 'demarginate' $[3-5,13]$.
Fig. 4 ANC PopPK/PD model. a Goodness-of-fit; b normalized prediction distribution error; c conditional weighted residuals; and $\mathbf{d}$ visual predictive check. $A N C$ absolute neutrophil count, $C W R E S$ conditional weighted residuals, $N P D E$ normalized prediction distribution error, PopPK/PD population pharmacokinetic/pharmacodynamic, $q w$ every week, $q 2 w$ every 2 weeks. In a, dashed blue lines indicate $y=x$, solid blue line indicates $y=0$, solid red line indicates tendency. In d, solid red line represents the median of observations; solid dashed lines represent the 2.5th and 97.5th percentiles of observations; and pink and gray areas represent the confidence intervals of the median and 5th and 95th percentiles of predictions

Further support that marginated neutrophils remain viable in case of infection is provided by previous studies, which have shown that neutrophils retain their functional capacity (such as respiratory burst or chemotaxis) under IL-6Ra blockade with tocilizumab [18, 19].

In clinical trials, ADAs were reported for $5.7 \%$ of patients treated with sarilumab $150 \mathrm{mg}$ every 2 weeks, $4.0 \%$ of those treated with sarilumab $200 \mathrm{mg}$ every 2 weeks, and $1.9 \%$ of those who received placebo $[8,9]$; NAbs were reported for $1.6 \%, 1.0 \%$, and $0.2 \%$ of patients, respectively $[8,9]$. No correlation was observed between ADA development and either loss of efficacy or the emergence of adverse reactions.

The prescribing guidance for subcutaneous sarilumab in the EU and the US is $200 \mathrm{mg}$ every 2 weeks, with adjustment to $150 \mathrm{mg}$ every 2 weeks for the management of neutropenia (as well as thrombocytopenia and liver enzyme elevations) $[8,9]$, due to the favorable benefit/risk profile for this regimen based on the results of the phase III clinical trials and subsequent long-term follow-up [3, 4, 8, 9, 13]. The current PopPK/ PD analysis contributes to the totality of evidence that supports this starting dose, with a numerically greater DAS28CRP reduction (and better potential to prevent irreversible joint damage) predicted at the median exposure of $200 \mathrm{mg}$ every 2 weeks compared with $150 \mathrm{mg}$ every 2 weeks. This analysis also supports the dose reduction scheme in the event of laboratory abnormalities (such as neutropenia, which is reversible) due to greater ANC reduction at a median exposure of $200 \mathrm{mg}$ every 2 weeks rather than $150 \mathrm{mg}$ every 2 weeks.

\section{Conclusions}

The PopPK/PD model presented here for DAS28-CRP and $\mathrm{ANC}$, in combination with previously reported exposure-response analysis conducted using data from phase III studies, is consistent with a subcutaneous sarilumab $200 \mathrm{mg}$ every 2 weeks dosing regimen, with a decrease to $150 \mathrm{mg}$ every 2 weeks in the event of laboratory abnormalities such as neutropenia. Four covariates were retained in the final models: body weight, baseline RF status, ACCP status, and concomitant methotrexate. There was no clinically meaningful influence of investigated covariates for either model, 
(a)
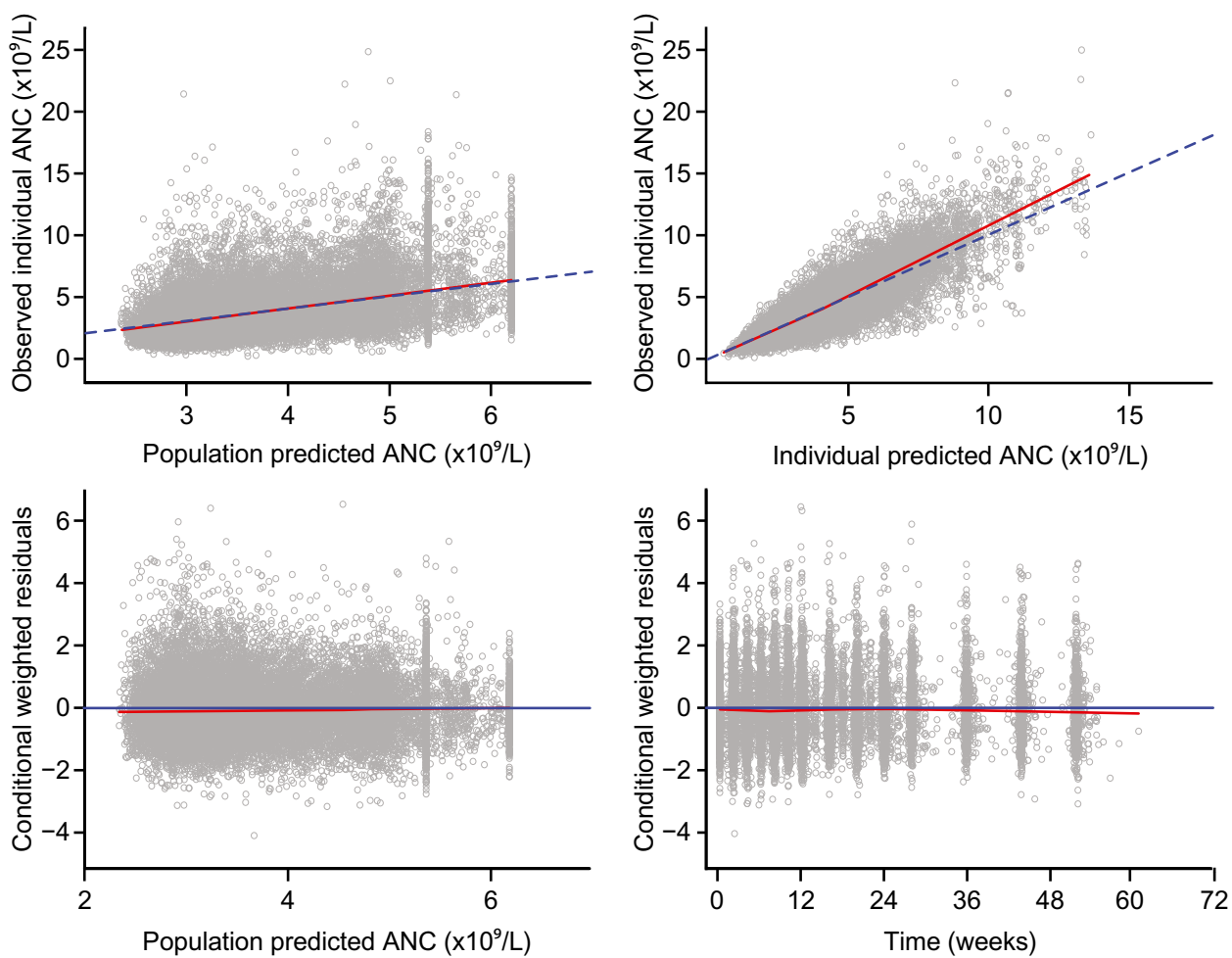

(b)
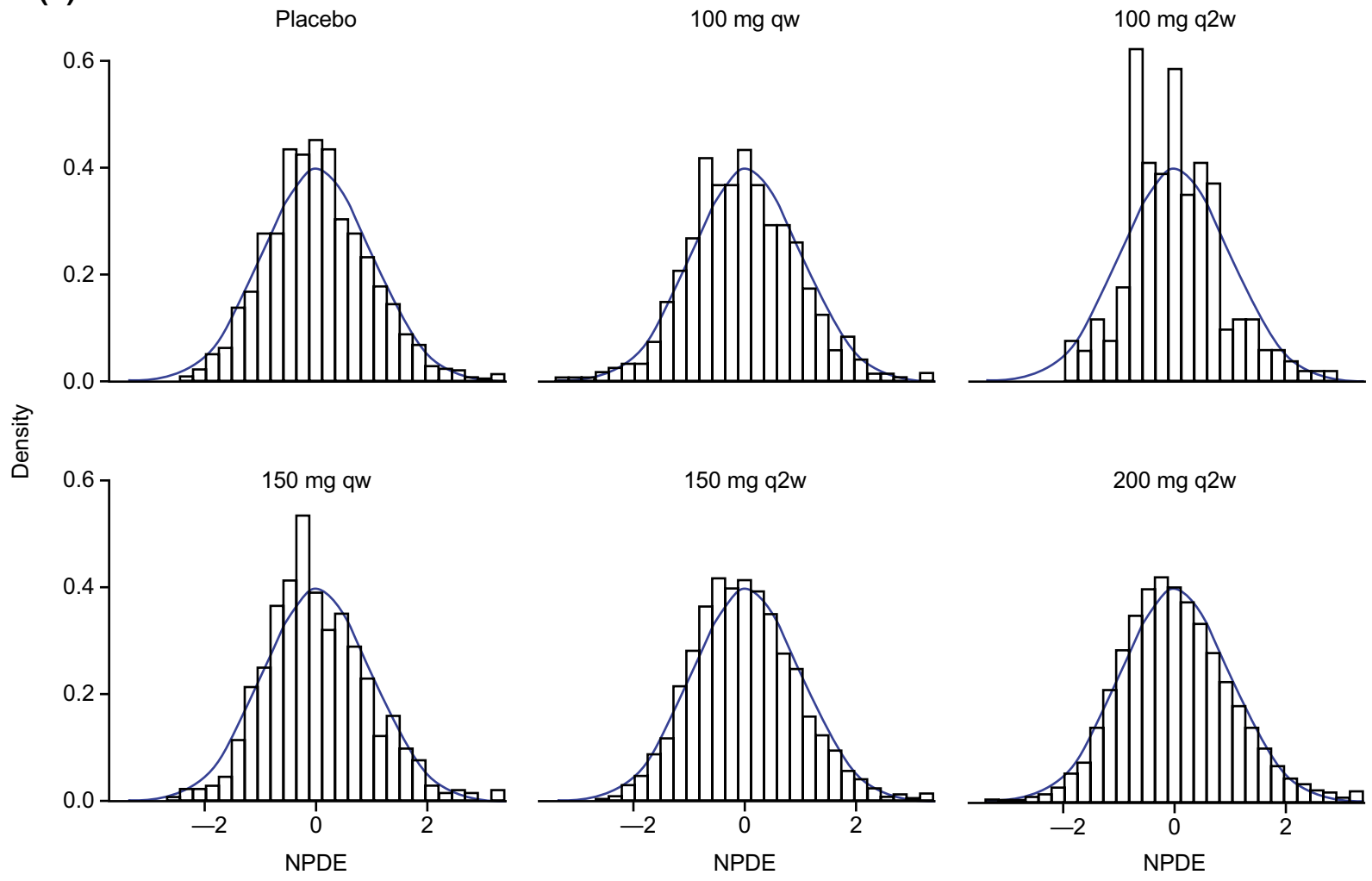

Normal density 
(c)
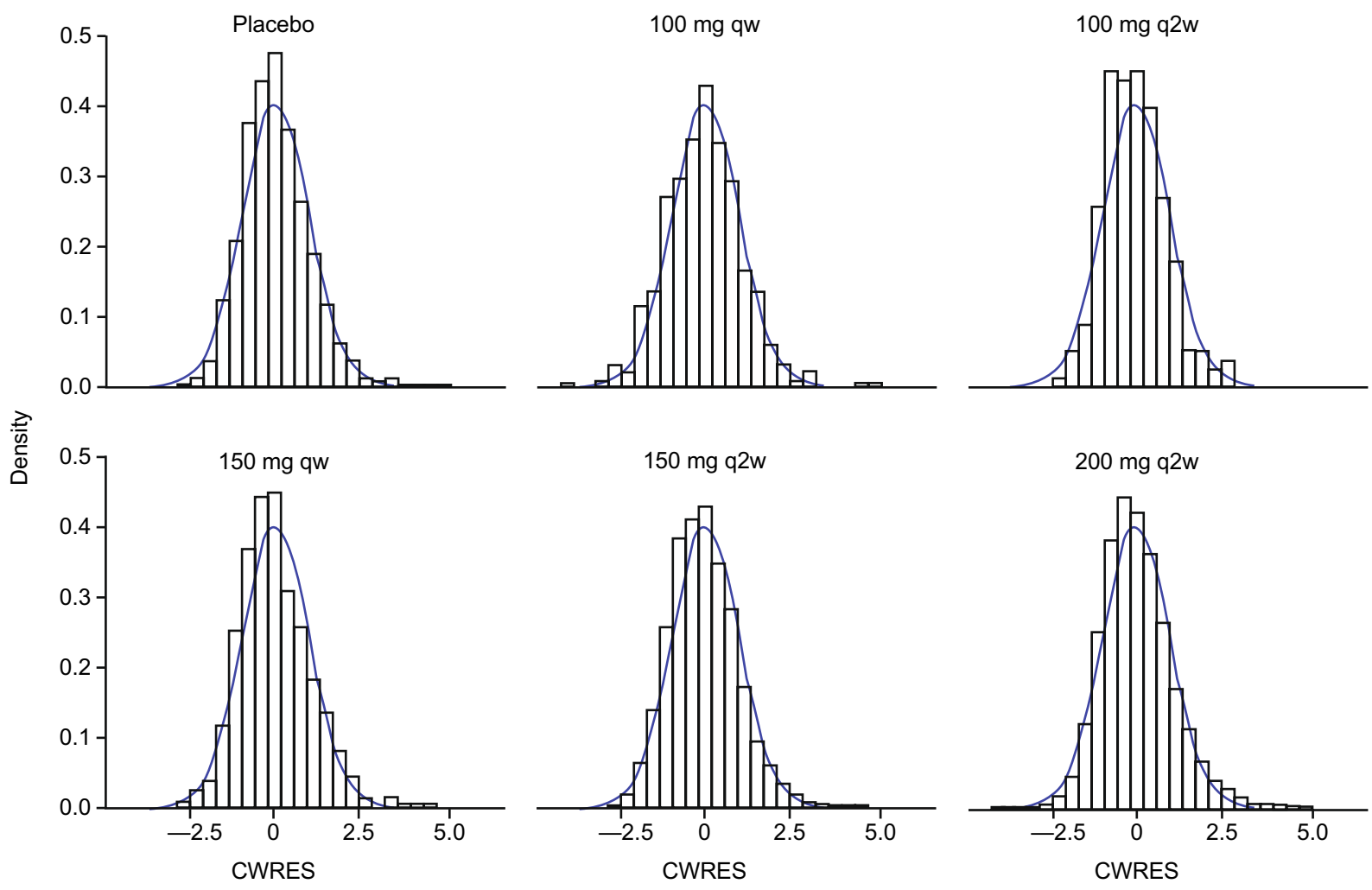

_ Normal density

(d)

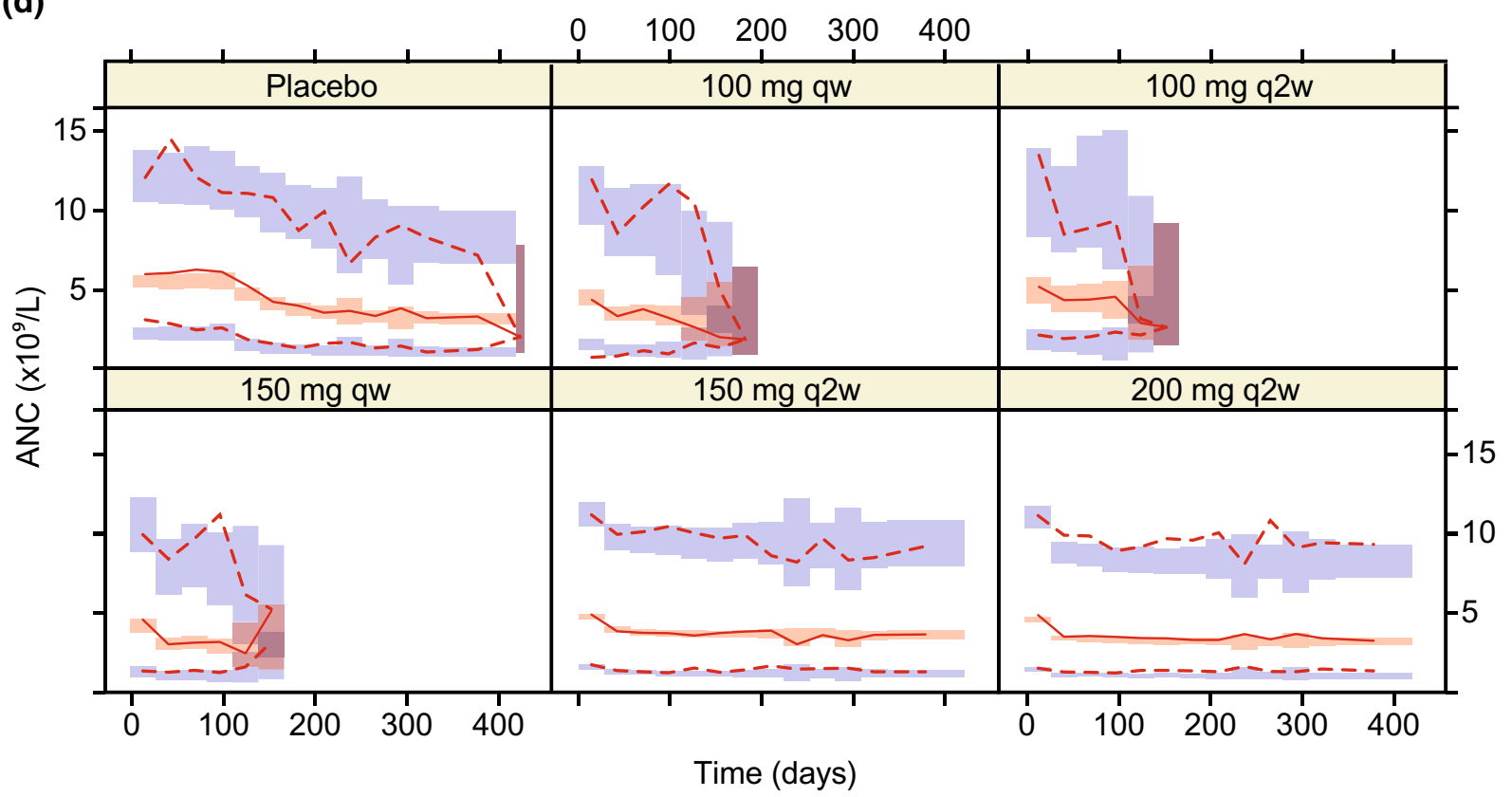

Fig. 4 (continued) 
Fig. 5 ANC time profiles (observed vs. model predicted). ANC absolute neutrophil count, $P 5$ 5th percentile, $P 95$ 95th percentile, $q 2 w$ every 2 weeks

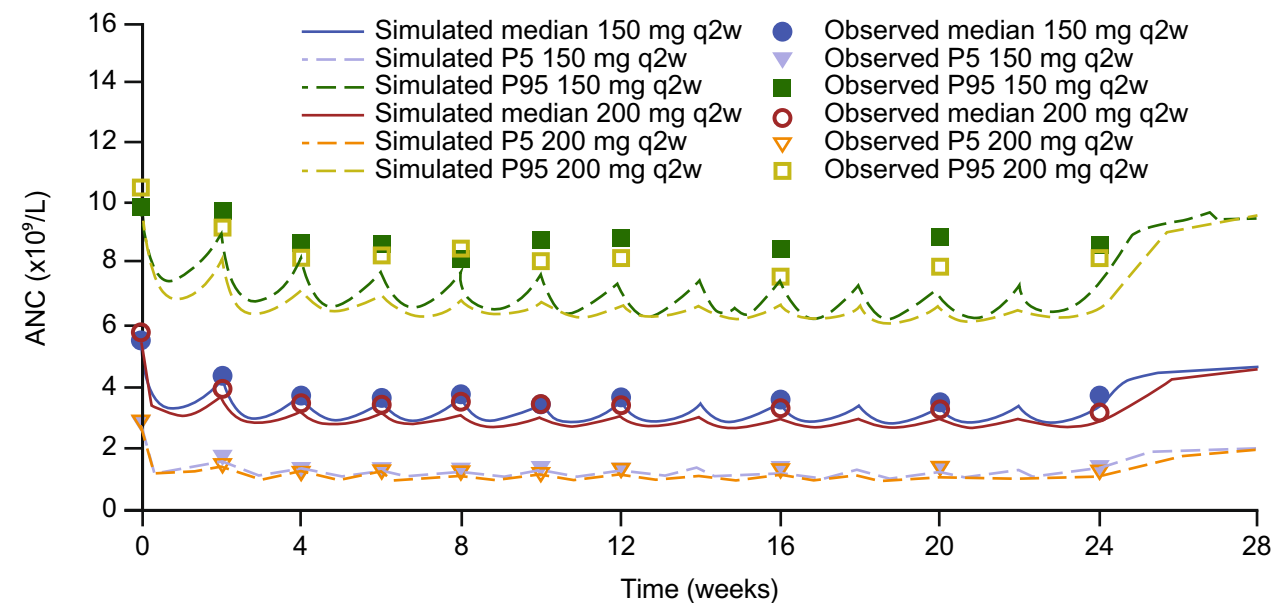

indicating dose adjustments are not required for these patient characteristics.

Acknowledgments Study funding and medical writing support (Tracey Lonergan, PhD, Adelphi Communications Ltd, Macclesfield, UK) were provided by Sanofi Genzyme (Cambridge, MA, USA) and Regeneron Pharmaceuticals, Inc (Tarrytown, NY, USA) in accordance with Good Publication Practice (GPP3) guidelines.

Funding This study was sponsored by Sanofi and Regeneron Pharmaceuticals, Inc. Editorial support for the development of this manuscript was provided by Tracey Lonergan, $\mathrm{PhD}$, Adelphi Communications, funded by Sanofi and Regeneron Pharmaceuticals, Inc.

Data availability Qualified researchers may request access to patientlevel data and related study documents, including the clinical study report, study protocol with any amendments, blank case report form, statistical analysis plan, and dataset specifications. Patient-level data will be anonymized and study documents will be redacted to protect the privacy of trial participants. Further details on Sanofi's data sharing criteria, eligible studies, and the process for requesting access can be found at: https://www.clinicalstudydatarequest.com

\section{Compliance with Ethical Standards}

Conflict of interest Christine Xu and Vanaja Kanamaluru are employees of Sanofi Genzyme and may hold stock and/or stock options in the company. Lei Ma was an employee of Sanofi Genzyme at the time of this work and may hold stock and/or stock options in the company. Anne Paccaly is an employee of Regeneron Pharmaceuticals, Inc and may hold stock and/or stock options in the company. None of the authors have any non-financial conflicts of interest to disclose.

Ethics approval For each individual study, all procedures were in accordance with the ethical standards of the relevant institutional and/ or national research committee and with the 1964 Declaration of Helsinki and its later amendments or comparable ethical standards.

Consent to participate Informed consent was obtained from all individual participants included in the individual studies.

Open Access This article is licensed under a Creative Commons Attribution-NonCommercial 4.0 International License, which permits any non-commercial use, sharing, adaptation, distribution and reproduction in any medium or format, as long as you give appropriate credit to the original author(s) and the source, provide a link to the Creative Commons licence, and indicate if changes were made. The images or other third party material in this article are included in the article's Creative Commons licence, unless indicated otherwise in a credit line to the material. If material is not included in the article's Creative Commons licence and your intended use is not permitted by statutory regulation or exceeds the permitted use, you will need to obtain permission directly from the copyright holder. To view a copy of this licence, visit $\mathrm{http} / / /$ creativecommons.org/licenses/by-nc/4.0/.

\section{References}

1. GBD 2017 Disease and Injury Incidence and Prevalence Collaborators. Global, regional, and national incidence, prevalence, and years lived with disability for 354 diseases and injuries for 195 countries and territories, 1990-2017: a systematic analysis for the Global Burden of Disease Study 2017. The Lancet. 2018;392:1789-858.

2. Yoshida Y, Tanaka T. Interleukin 6 and rheumatoid arthritis Biomed Res Int. 2014;2014:698313.

3. Fleischmann R, van Adelsberg J, Lin Y, Castelar-Pinheiro GD, Brzezicki J, Hrycaj P, et al. Sarilumab and nonbiologic diseasemodifying antirheumatic drugs in patients with active rheumatoid arthritis and inadequate response or intolerance to tumor necrosis factor inhibitors. Arthr Rheumatol. 2017;69:277-90.

4. Genovese MC, Fleischmann R, Kivitz AJ, Rell-Bakalarska M, Martincova R, Fiore S, et al. Sarilumab plus methotrexate in patients with active rheumatoid arthritis and inadequate response to methotrexate: results of a phase III study. Arthr Rheumatol. 2015;67:1424-37.

5. Burmester GR, Lin Y, Patel R, van Adelsberg J, Mangan EK, Graham NM, et al. Efficacy and safety of sarilumab monotherapy versus adalimumab monotherapy for the treatment of patients with active rheumatoid arthritis (MONARCH): a randomised, double-blind, parallel-group phase III trial. Ann Rheum Dis. 2017;76:840-7.

6. Ruderman EM. Rheumatoid arthritis: IL-6 inhibition in RA-deja vu all over again? Nat Rev Rheumatol. 2015;11:321-2.

7. Scott LJ. Sarilumab: first global approval. Drugs. 2017;77:705-12.

8. Kevzara. US prescribing information. 2018. https://www.accessdata.fda.gov/drugsatfda_docs/label/2017/761037s000lbl.pdf. Cited Sep 2019. 
9. Kevzara. Summary of product characteristics. 2019. https://www. medicines.org.uk/emc/medicine/33836. Cited 12 Aug 2019.

10. Sebastien B, Sun S, Rigaux-Lampe C, Quan H, Paccaly A, Lin $\mathrm{Y}$, et al. Benefit/risk assessment of sarilumab using exposure/ response modeling of key efficacy and safety endpoints in patients with rheumatoid arthritis. American conference on pharmacometrics (ACoP) 10, Vol 1; 2019; Orlando, FL. ISSN:2688-3953.

11. Meng Z, Sebastien B, Sun S, Rigaux-Lampe C, Paccaly A, Xu C, et al. Exposure/response modeling for sarilumab dose regimens benefit/risk assessment. Population Approach Group in Europe (PAGE) meeting 2017: Budapest; 2017. p. 7285.

12. Suwa T, Hogg JC, English D, Van Eeden SF. Interleukin-6 induces demargination of intravascular neutrophils and shortens their transit in marrow. Am J Physiol Heart Circ Physiol. 2000;279:H2954-H29602960.

13. Fleischmann R, Lin Y, John GS, Heijde Dvd, Qiu C, GomezReino JJ, et al. Long-term safety with sarilumab plus conventional synthetic disease-modifying antirheumatic drugs and sarilumab monotherapy in rheumatoid arthritis: an integrated analysis with 9,000 patient-years of follow-up. Ann Rheum Dis. 2019;78 Suppl 2:1130-1.
14. Xu C, Su Y, Paccaly A, Kanamaluru V. Population pharmacokinetics of sarilumab in patients with rheumatoid arthritis. Clin Pharmacokinet. 2019;58:1455-67.

15. Gibiansky L, Frey N. Linking interleukin-6 receptor blockade with tocilizumab and its hematological effects using a modeling approach. J Pharmacokinet Pharmacodyn. 2012;39:5-16.

16. Kovalenko P, Paccaly A, Boyapati A, Xu C, Davis J, DiCioccio T. Pharmacodynamic (PD) model of neutrophil margination to describe transient effect of single-dose sarilumab on absolute neutrophil count (ANC) in patients with rheumatoid arthritis (RA). 9th American conference on pharmacometrics, Fort Lauderdale, FL; 2017.

17. Ishii T, Sato Y, Munakata Y, Kajiwara M, Takahashi Y, Anwar F, et al. AB0472 pharmacodynamic effect and safety of single-dose sarilumab sc or tocilizumab iv or sc in patients with rheumatoid arthritis (RA). Ann Rheum Dis. 2018;2018:1397-8.

18. Wright HL, Cross AL, Edwards SW, Moots RJ. Effects of IL-6 and IL-6 blockade on neutrophil function in vitro and in vivo. Rheumatology (Oxford). 2014;53:1321-31.

19. Lok LSC, Farahi N, Juss JK, Loutsios C, Solanki CK, Peters AM, et al. Effects of tocilizumab on neutrophil function and kinetics. Eur J Clin Invest. 2017;47:736-45. 